\title{
The Free Particle in Deformed Special Relativity
}

\author{
Florian Girelli, ${ }^{1, *}$ Tomasz Konopka, ${ }^{1,2, \dagger}$ Jerzy Kowalski-Glikman, ${ }^{3, \ddagger}$ and Etera R. Livine ${ }^{1,4, \S}$ \\ ${ }^{1}$ Perimeter Institute, 31 Caroline Street North Waterloo, Ontario, Canada N2L 2 Y5 \\ ${ }^{2}$ University of Waterloo, 200 University Ave. West, Waterloo, ON, N2L 3G1, Canada \\ ${ }^{3}$ Institute for Theoretical Physics, University of Wroctaw, Pl. Maxa Borna 9, Pl-50-204 Wroctaw, Poland \\ ${ }^{4}$ Laboratoire de Physique, ENS Lyon, CNRS UMR 5672, 46 Allée d'Italie, 69364 Lyon Cedex 07
}

\begin{abstract}
The phase space of a classical particle in DSR contains de Sitter space as the space of momenta. We start from the standard relativistic particle in five dimensions with an extra constraint and reduce it to four dimensional DSR by imposing appropriate gauge fixing. We analyze some physical properties of the resulting theories like the equations of motion, the form of Lorentz transformations and the issue of velocity. We also address the problem of the origin and interpretation of different bases in DSR.
\end{abstract}

PACS numbers:

\section{INTRODUCTION}

In many situations, it is of interest to discuss phase spaces with curvature. Examples of models with curved position spaces abound, but models with curved momentum space can also be quite interesting [1-4]. One of their common features is that they are all closely related to non-commutative geometries (see eg. [5]). Some new investigations suggest that these models might be relevant to quantum gravity. For instance, it has been found that a particle coupled to gravity in three dimensions has a curved, maximally symmetric momentum space $[6,7]$. Similar properties have been argued to hold in four dimensions as well [8]. As another example, Deformed Special Relativity (DSR) [9-13], which has been argued to represent an effective low-energy description of quantum gravity, also implements momentum space as de Sitter $[14,15]$ or anti de Sitter [16].

In usual relativistic physics, the phase space of a particle consists of the position space and the momentum space both of which are four-dimensional Minkowski spaces. One also introduces an action which determines the canonical coordinates on the phase space and their dynamics. On the other hand, in DSR, kinematics and dynamics are usually defined separately. One first specifies a deformed symplectic form on the phase space which reflects the fact that the momentum space is de Sitter. The dynamics is then determined by the symmetries and the Hamiltonian is constructed from the Casimir of the Poincare group. The issue that we address here is to determine an action principle for DSR which generates both

\footnotetext{
* Electronic address: fgirelli@perimeterinstitute.ca

$\dagger$ Electronic address: tkonopka@perimeterinstitute.ca

${ }^{\ddagger}$ Electronic address: jurekk@ift.uni.wroc.pl

$\S$ Electronic address: elivine@perimeterinstitute.ca
}

the symplectic form and the dynamics.

The next section is devoted to description of the standard relativistic particle, with special emphasis put on the way to introduce time and gauge fixing.

In section III, we explain how to reduce the relativistic particle in a five-dimensional Minkowski space to a particle with a four-dimensional curved de Sitter space of momenta. We constrain the 10d flat phase space with an appropriate gauge fixing to reduce it to a $8 \mathrm{~d}$ phase space with a de Sitter energy-momentum sector. Since our starting point is the phase space with natural coordinates in 10 dimensions, choosing a gauge fixing condition is equivalent to defining some particular coordinates in the resulting 8 dimensional phase space. We take the reduced phase space to define the single particle in DSR.

In sections IV and V, we follow the gauge fixing procedure and provide the explicit gauge fixing conditions leading to the Snyder and the bicrossproduct bases (we deal with these two bases because they are the most frequently used DSR models.) This shows how the traditional formulations of DSR can be recovered from a single $5 \mathrm{~d}$ action principle. We then discuss the DSR physics in these two bases, equations of motion, speed-momentum relation, boosts and length contraction. In section VI, it is pointed out that another basis found in the literature does not fit in the gauge-fixing scheme.

In section VII, we discuss the description of manyparticle systems and the definition of a total momentum. In the final section, we argue that physical predictions should be independent of the choice of gauge fixing condition while the choice of particular $4 \mathrm{~d}$ coordinates should correspond to the choice of specific measurement protocols for $x$ and $p$ in the context of general relativity providing us with a fluctuating space-time geometry.

We use capital letters $X$ and $P$ to denote coordinates in the initial phase space and small letters $x$ and $p$ to denote coordinates on the reduced phase space. Capital Latin indices refer to 5 dimensional spacetime, Greek to 
4 dimensional spacetime, while small Latin to 3 dimensional space. Contractions on $4 \mathrm{~d}$ variables, such as $p^{2}$ and $x \cdot p$, are all defined using a metric $\eta_{\mu \nu}=\operatorname{diag}(+---)$.

\section{A REVIEW OF THE RELATIVISTIC PARTICLE}

\section{A. The Action and the Legendre transform}

Let us consider the free relativistic spinless particle of mass $m$ in 4 dimensions.

In the Lagrangian formulation, the action is given by the line element in the flat metric $\eta=(+---)$

$$
\mathcal{S} \equiv m \int d s=m \int d \tau \sqrt{\dot{x}^{\mu} \dot{x}_{\mu}}=\int d \tau \mathcal{L},
$$

which we parametrize in terms of an arbitrary time parameter. The Euler-Lagrange equations specify the symplectic form and phase space structure. The canonical momentum is defined as

$$
p_{\mu}=\frac{\partial \mathcal{L}}{\partial \dot{x}^{\mu}}=m \frac{\dot{x}_{\mu}}{\sqrt{\dot{x}^{\mu} \dot{x}_{\mu}}}
$$

and the Poisson bracket on the phase space is canonical

$$
\left\{x_{\mu}, p_{\nu}\right\}=\eta_{\mu \nu}
$$

Applying the Legendre transform, we check that $\mathcal{L}$ is simply equal to $\dot{x}^{\mu} p_{\mu}$, so that the Hamiltonian vanishes. Nevertheless, the momentum $p_{\mu}$ satisfies $\mathcal{H}=0$ with

$$
\mathcal{H}=p^{2}-m^{2},
$$

which we call the Hamiltonian constraint. Finally the free particle action is written in the first order formalism

$$
\mathcal{S}=\int d \tau\left(\dot{x}^{\mu} p_{\mu}-\lambda \mathcal{H}\right),
$$

where $\lambda$ is a Lagrange multiplier. Note that while the action (1) cannot describe massless particles, the action (5) can.

The latter action (5) in the hamiltonian formalism can be taken as a starting point for the study the relativistic particle as an alternative to (1). The constraint $\mathcal{H}$ generates a gauge symmetry whose flow defines the equations of motion:

$$
\begin{aligned}
\delta x_{\mu} & =\left\{x_{\mu}, \lambda \mathcal{H}\right\}=2 \lambda p_{\mu}, \\
\delta p_{\mu} & =\left\{p_{\mu}, \lambda \mathcal{H}\right\}=0 .
\end{aligned}
$$

Enforcing the mass-shell condition $p_{\mu} p^{\mu}=m^{2}$ fixes the value of the Lagrange multiplier

$$
\lambda=\frac{1}{2 m} \sqrt{\delta x_{\mu} \delta x^{\mu}}
$$

so that the momentum is

$$
p_{\mu}=m \frac{\delta x_{\mu}}{\sqrt{\delta x_{\nu} \delta x^{\nu}}}=m \frac{\dot{x}_{\mu}}{\sqrt{\dot{x}_{\nu} \dot{x}^{\nu}}} .
$$

Inserting this expression in the action (5), we recover the original action (1) defined as the line element.

\section{B. Introducing Time}

A time variable is a phase space function that does not commute with the Hamiltonian (constraint).

The time parameter that is usually chosen is $t=x_{0}$. As seen in (6), $x_{0}$ does not commute with $\lambda \mathcal{H}$. Then the equations of motion of the other variables in terms of the chosen time are obtained by the flow of the Hamiltonian constraint. Indeed, since $\left\{\lambda \mathcal{H}, x_{\mu}\right\}=-2 \lambda p_{\mu} \neq 0$ and $\left\{\lambda \mathcal{H}, p_{\mu}\right\}=0$, we have

$$
\dot{x}_{i} \equiv \frac{\delta x_{i}}{\delta x_{0}}=\frac{p_{i}}{p_{0}}, \quad \dot{p}_{i}=0 .
$$

The first equation defines a notion of speed $v_{i}=\dot{x}_{i}=$ $p_{i} / p_{0}$.

Substituting $t=x_{0}$ into the action (5) gives $S=$ $\int d t\left[\dot{x}_{i} p_{i}-p_{0}-\lambda\left(p^{2}-m^{2}\right)\right]$. Solving the constraint, we get $p_{0}= \pm \sqrt{\vec{p}^{2}+m^{2}}$. Therefore our action reduces to

$$
S=\int d t\left(\dot{x}_{i} p_{i}-H\right)
$$

where the Hamiltonian now does not vanish and is $H=$ $\pm \sqrt{\vec{p}^{2}+m^{2}}$, the sign depending on whether we consider the positive or negative energy branch.

Another "canonical" choice of time variable is $t=$ $D / m$, where $D \equiv x_{\mu} p^{\mu}$ is the dilatation operator. This is an acceptable time variable as $D$ does not commute with $\mathcal{H}$,

$$
\left\{\lambda \mathcal{H}, \frac{D}{m}\right\}=-2 \lambda \frac{p^{2}}{m} \neq 0 .
$$

Now we get the relativistic velocity

$$
\dot{x}_{\mu} \equiv m \frac{\delta x_{\mu}}{\delta D}=m \frac{p_{\mu}}{p^{2}}=\frac{p_{\mu}}{m} .
$$

If we want to make a time-space splitting with $D$ as the time, we need to identify space variables which commute with $D$ so that $D$ can be considered as establishing a legitimate clock system. The standard space coordinates $x_{i}$ 's do not commute with $D$. Nevertheless, we can define normalized space variables $y_{i} \equiv\left(x_{i} \sqrt{p^{2}}\right) / m$, which do commute with our chosen time $D / m$ and which are 
equal to the standard space coordinates on-shell. Their velocities are

$$
\dot{y}_{i}=\frac{p_{i}}{\sqrt{p^{2}}}
$$

and reduce to $p_{i} / m=\gamma v_{i}$ on the mass-shell. However, these speeds are not bounded by the speed of light, contrary to (7), and so cannot be regarded as physical speeds. This shows that we can choose theoretically any time variable to describe the evolution of the relativistic particle, the standard time coordinate $x_{0}$ remains the physical coordinate time.

Actually, thinking of $D / m$ as the proper time along the trajectory of the particle, i.e as the projection of the position vector $x_{\mu}$ along the particle momentum $p_{\mu}$, it is natural to introduce the transversal position variables

$$
\widetilde{x}_{\mu}=\left(\eta_{\mu \nu}-\frac{p_{\mu} p_{\nu}}{p^{2}}\right) x_{\nu}
$$

which are orthogonal to $p_{\mu}, p_{\mu} \widetilde{x}^{\mu}=0$. Although $\widetilde{x}$ is defined as a 4 -vector, it has only three (independent) components. And the vector $(D / m, \widetilde{x})$ defines a coordinate system -the parallel and transverse projection of the 4-vector $x_{\mu}$ along the momentum $p_{\mu}$. The $\widetilde{x}_{\mu}$ 's do not commute with the dilatation time $D / m$, but we can rescale them by the factor $\sqrt{p^{2}} / \mathrm{m}$. The interesting feature of this choice of space coordinates is that the $\widetilde{x}_{\mu}$ do commute with the Hamiltonian constraint. They are actually Dirac observables and they do not evolve (in time): the gauge-fixed Hamiltonian describing the evolution of the space coordinates $\widetilde{x}$ the particle with respect of the time $D / m$ vanishes.

It is possible to generalize this remark and construct position operators for the relativistic particle which are Dirac observables. They would commute with the Hamiltonian and be constant of the motion. Actually, all Dirac observables of the relativistic particle are generated by the Poincaré generators $p_{\mu}$ and $j_{\mu \nu}=x_{\mu} p_{\nu}-p_{\mu} x_{\nu}$. From these, one can construct position operators corresponding to the two previous choices of time $x_{0}$ and $D$ :

$$
X_{\mu}^{\left(x_{0}\right)}(t)=\frac{j_{\mu \nu} v^{\nu}+t p_{\mu}}{p \cdot v}=x_{\mu}+\frac{p_{\mu}}{p_{0}}\left(t-x_{0}\right),
$$

with $v=(1,0,0,0)$, and

$$
X_{\mu}^{(D)}(t)=\frac{j_{\mu \nu} p^{\nu}+t p_{\mu}}{p^{2}}=x_{\mu}+\frac{p_{\mu}}{p^{2}}(t-D)=\widetilde{x}_{\mu}+\frac{p_{\mu}}{p^{2}} t .
$$

These are relational observables indicating the evolved positions at the time $t$ (with respect to the chosen clock $x_{0}$ or $\left.D\right)$. A detailed analysis of the properties and quantization of these Dirac observables is under investigation [18].

\section{Gauge Fixing and the Dirac bracket}

The choice of a time variable can be regarded as an explicit gauge fixing that breaks the symmetry of the action under time reparametrization. After gauge fixing, the symplectic form on the reduced phase space is given by the Dirac bracket. Given a constraint $\mathcal{H}$ and a gauge fixing condition $\mathcal{C}$ such that $\{\mathcal{C}, \mathcal{H}\} \neq 0$, the Dirac bracket is defined as

$$
\begin{aligned}
\{\phi, \psi\}_{D}= & \{\phi, \psi\}-\{\phi, \mathcal{C}\}\left(\frac{1}{\{\mathcal{H}, \mathcal{C}\}}\right)\{\mathcal{H}, \psi\} \\
& -\{\phi, \mathcal{H}\}\left(\frac{-1}{\{\mathcal{H}, \mathcal{C}\}}\right)\{\mathcal{C}, \psi\} .
\end{aligned}
$$

For the standard time choice $\mathcal{C}=x_{0}-t$, where $t$ is a free parameter, we obtain

$$
\begin{aligned}
\left\{x_{0}, p_{0}\right\}_{D} & =0, \\
\left\{x_{i}, p_{0}\right\}_{D} & =-v_{i}, \\
\left\{x_{\mu}, x_{\nu}\right\}_{D} & =0 .
\end{aligned}
$$

Note that $p_{0}$ generates the usual Hamiltonian flow under the Dirac bracket and is actually the true physical Hamiltonian dictating the time evolution with respect to the clock $x_{0}$.

The important remark linking the Dirac observables to the Dirac bracket defining the symplectic structure on the reduced phase space is the following equalities:

$$
\begin{aligned}
& \left\{x_{\mu}, p_{\nu}\right\}_{D}=\left\{X_{\mu}^{\left(x_{0}\right)}(t), p_{\nu}\right\}, \\
& \left\{x_{\mu}, x_{\nu}\right\}_{D}=\left\{X_{\mu}^{\left(x_{0}\right)}(t), X_{\nu}^{\left(x_{0}\right)}(t)\right\},
\end{aligned}
$$

for any fixed parameter $t$. These formula summarize the explicit link between the gauge fixing procedure allowing us to generate a time evolution and the algebra of Dirac observables which are constants of motion.

In the case of the Lorentz invariant gauge fixing defined by the dilatation $\mathcal{C}=D / m-t$, we compute

$$
\begin{aligned}
& \left\{x_{\mu}, p_{\nu}\right\}_{D}=\eta_{\mu \nu}-\frac{p_{\mu} p_{\nu}}{m^{2}}, \\
& \left\{x_{\mu}, x_{\nu}\right\}_{D}=-\frac{j_{\mu \nu}}{m^{2}} .
\end{aligned}
$$

And once again we obtain the following equalities:

$$
\begin{aligned}
\left\{x_{\mu}, p_{\nu}\right\}_{D} & =\left\{X_{\mu}^{(D)}(t), p_{\nu}\right\} \\
\left\{x_{\mu}, x_{\nu}\right\}_{D} & =\left\{X_{\mu}^{(D)}(t), X_{\nu}^{(D)}(t)\right\} .
\end{aligned}
$$

\section{DEFORMED SPECIAL RELATIVITY}

\section{A. A 5d Action Principle}

We now turn to the relativistic particle in the context of Deformed Special Relativity (DSR). The idea behind 
DSR is that the four-dimensional momentum space of the particle is curved. More precisely, we choose a constant positive curvature i.e. the de Sitter space. This is implemented as a constraint in the action.

The full action for the particle in the Hamiltonian formalism is

$$
S_{5 d}=\int d \tau\left[\dot{X}_{A} P^{A}-\Lambda \mathcal{H}_{5 d}-\lambda \mathcal{H}_{4 d}\right] .
$$

The coordinates $X_{A}$ and $P^{A}$ form a ten dimensional phase space with the usual symplectic structure $\left\{X_{A}, P_{B}\right\}=\eta_{A B}$. The first constraint

$$
\mathcal{H}_{5 d}=P_{A} P^{A}+\kappa^{2}
$$

imposes that the physical $4 \mathrm{~d}$ momentum effectively lives on the four-dimensional de Sitter space $\mathrm{SO}(4,1) / \mathrm{SO}(3,1)$. The second constraint

$$
\mathcal{H}_{4 d}=P_{4}-M
$$

is identified as the mass-shell constraint $\mathcal{H}$ in (4) and generates the Hamiltonian flow on the reduced phase space. The mass $M$ is a function of the rest mass $m$ and the universal mass scale $\kappa$. We will show in the following section how $\mathcal{H}_{4 d}$ becomes the Hamiltonian constraint of the deformed relativistic particle and how it is actually a $\kappa$-dependent deformation of the traditional $p^{2}-m^{2}$. Actually, instead of $\mathcal{H}_{4 d}=P_{4}-M$, we could take $\mathcal{H}_{4 d}=V^{A} P_{A}-M$ for any (unit) 5 d space-like vector.

We remark that the structure of this action is very similar to the reformulation of general relativity as a perturbed BF theory with a $\mathrm{SO}(4,1)$ gauge symmetry studied by Freidel and Starodubtsev in [21]. Indeed, the following action has the Einstein equations as classical equations of motion:

$$
\begin{aligned}
S_{F S}[B, A]= & \int B^{I J} \wedge F_{I J}[A]-\frac{\beta}{2} B^{I J} \wedge B_{I J} \\
& -\frac{\alpha}{4} \int V^{M} \epsilon_{I J K L M} B^{I J} \wedge B^{K L},
\end{aligned}
$$

where $V^{M}$ is a unit vector and $I, . ., M=0 . .4$ are $\mathfrak{s o}(4,1)$ indices. The coupling constants $\alpha$ and $\beta$ are related to the cosmological constant and the Immirzi parameter (which is related to $\mathrm{CP}$ violation). The kinematical term of the action is the topological BF gauge theory. The second term is a $\mathrm{SO}(4,1)$-invariant mass term which introduces the cosmological constant. The third term explicitly breaks the $\mathrm{SO}(4,1)$ gauge symmetry down to a $\mathrm{SO}(3,1)$ gauge symmetry and allows to recover general relativity as a sector of the topological BF theory. We believe that the similarity between this reformulation of general relativity as a $\mathrm{SO}(4,1)$ gauge theory and the $5 \mathrm{~d}$ action for deformed special relativity might point to the fact that our $5 \mathrm{~d}$ action would actually describe the dynamics of (test) particles in this framework.

The symmetries of the action are generated by those operators that commute with both of the constraints. It is straightforward to check that the 4 dimensional angular momentum operators,

$$
J_{\mu \nu}=X_{\mu} P_{\nu}-X_{\nu} P_{\mu},
$$

commute with the two constraints. The $J_{\mu \nu}$ 's together with the $P_{\mu}$ 's are the generators of the ten-dimensional Poincaré Lie algebra. We also refer to these operators as the spacetime boosts $N_{i}=J_{0 i}$ and the rotations $M_{i j}=$ $J_{i j}$.

\section{B. Gauge Fixing down to DSR's}

The proposed action $S_{5 d}$ is defined independently of any choice of "basis" or coordinate system on de Sitter. Standard theories of DSR are then to be obtained through a gauge fixing of the $5 \mathrm{~d} \kappa$-shell constraint $\mathcal{H}_{5 d}$.

More precisely, a gauge fixing is defined as a phase space function $\mathcal{C}$ which does not commute with $\mathcal{H}_{5 d}$ :

$$
\left\{\mathcal{H}_{5 d}, \mathcal{C}\right\} \neq 0 .
$$

The additional constraint $\mathcal{C}=0$ turns $\mathcal{H}_{5 d}$ into a second class constraint and imposing $\mathcal{H}_{5 d}=\mathcal{C}=0$ will reduce the initial 10-dimensional phase space to a more traditional 8-dimensional phase space for a relativistic particle. The symplectic structure induced on the reduced phase space is the Dirac bracket defined as previously by:

$$
\begin{aligned}
\{\phi, \psi\}_{D}= & \{\phi, \psi\}-\{\phi, \mathcal{C}\}\left(\frac{1}{\{\mathcal{H}, \mathcal{C}\}}\right)\{\mathcal{H}, \psi\} \\
& -\{\phi, \mathcal{H}\}\left(\frac{-1}{\{\mathcal{H}, \mathcal{C}\}}\right)\{\mathcal{C}, \psi\} .
\end{aligned}
$$

A choice of proper coordinates on the reduced phase space are $\left(x_{\mu}, p_{\mu}\right)$ that commute with both $\mathcal{H}_{5 d}$ and $\mathcal{C}$ :

$$
\left\{x_{\mu}, \mathcal{H}_{5 d}\right\}=\left\{x_{\mu}, \mathcal{C}\right\}=\left\{p_{\mu}, \mathcal{H}_{5 d}\right\}=\left\{p_{\mu}, \mathcal{C}\right\}=0 .
$$

Their Dirac bracket with any phase space function is exactly equal to their Poisson bracket with that same function:

$$
\forall F(X, P),\{x, F\}_{D}=\{x, F\}, \quad\{p, F\}_{D}=\{p, F\} .
$$

We usually choose $p_{\mu}$ as functions of solely the $5 \mathrm{~d}$ momentum coordinates $P_{A}$ while the $x_{\mu}$ are a mixture of $X_{A}$ and $P_{A}$. The $p_{\mu}$ 's are a choice of coordinate system of the 4-dimensional De Sitter space, while the $x_{\mu}$ are a choice of generators of the translations on this same De Sitter 
space. Usual DSR theories are formulated through such a choice of coordinates $\left(x_{\mu}, p_{\mu}\right)$, which is called a choice of basis. Here we identify the ambiguity as the choice of a gauge fixing condition.

Moreover, now having ten phase space functions $\mathcal{H}_{5 d}, \mathcal{C}, x_{\mu}, p_{\mu}$, we can invert the relation between the $4 \mathrm{~d}$ coordinates and the $5 \mathrm{~d}$ coordinates and express the $X_{A}$ and $P_{A}$ in terms of $x_{\mu}, p_{\mu}$ and $\mathcal{H}_{5 d}, \mathcal{C}$. Since $\mathcal{H}_{5 d}$ and $\mathcal{C}$ commutes with the $x$ 's and $p$ 's, we can fix them at some particular values and express $X, P$ in term of $x, p$ and the real parameters $\kappa$ and $T$. Actually $\mathcal{C}$ is not needed to express the $5 \mathrm{~d}$ momentum coordinates $P_{A}$ which are a function of $p_{\mu}$ and $\kappa$ only; $\mathcal{C}$ is necessary, however, to express the $5 \mathrm{~d}$ coordinates $X_{A}$ in terms of the $4 \mathrm{~d}$ coordinates $x_{\mu}$. Thus, the condition $\mathcal{C}$ is crucial to understanding the "meaning" of the fifth space-time coordinate $X_{4}$.

Finally, we have the interesting property that any phase space function $\varphi(X, P)$ commuting with both the constraint $\mathcal{H}_{5 d}$ and the gauge fixing condition $\mathcal{C}$ is actually a function of solely the $x, p$ 's. In particular, this implies that the $x, p$ variables form a closed algebra under the Poisson bracket and define a true $4 \mathrm{~d}$ phase space without having $5 \mathrm{~d}$ variables appearing in their commutation relations. This also implies that it should be possible to quantize the DSR particle as a $4 \mathrm{~d}$ system, without necessarily having to deal with the $5 \mathrm{~d}$ variables. Nevertheless, we feel that a proper quantization should be done at the fully five-dimensional context without introducing any particular gauge fixing.

Since the different DSR theories can be seen as particular gauge fixing of this $5 \mathrm{~d}$ action, we expect that the physical predictions of the theory should be independent from the gauge fixing choice and depend solely on the details of the $5 \mathrm{~d}$ action. Moreover, as we know that gauge fixing and quantization do not commute, we expect that the quantization of the relativistic particle in DSR should be done from the $5 \mathrm{~d}$ point of view and not in a particular basis of a DSR theory.

\section{The 5d interpretation of DSR}

We note that the $5 \mathrm{~d}$ action (15) can be rewritten in a suggestive manner as

$$
S_{5 d}=S_{4 d}+S_{4}-S_{I}
$$

Here $S_{4 d}$ is the standard (undeformed) relativistic particle,

$$
S_{4 d}=\int d \tau\left[\dot{X}_{\mu} P^{\mu}-\lambda\left(P_{\mu} P^{\mu}-\widetilde{M}^{2}\right)\right],
$$

with a renormalized mass $\widetilde{M}^{2}=M^{2}-\kappa^{2}$. This works if and only if $M>\kappa . S_{4}$ is an extra degree of freedom,

$$
S_{4}=-\int d \tau \dot{X}_{4} P_{4},
$$

and $S_{I}$ is an interaction term enforcing the energy conservation of the coupled system of the particle and the extra degree of freedom,

$$
S_{I}=\Lambda \int d \tau\left(P_{\mu} P^{\mu}-P_{4}^{2}+\kappa^{2}\right) .
$$

The extra degree of freedom comes in with an negative energy and thus resembles a conformal mode. This suggests to consider DSR as describing a standard relativistic particle coupled to some effective degree of freedom, which could come from some effective description of (quantum) gravity. This point of view will be investigated in more details in future work.

\section{SNYDER BASIS}

The gauge fixing condition $\mathcal{C}$ defining the Snyder basis is the $5 \mathrm{~d}$ dilatation

$$
\mathcal{C}=\mathcal{D}-T=X_{A} P^{A}-T
$$

where $T$ is an arbitrary real parameter. In the following, we study the reduced phase space of the particle, after the gauge is imposed, as well as the equations of motion and the kinematics of the particle in this gauge.

\section{A. Reduced phase space}

Let us introduce the following four-dimensional position and momentum

$$
\begin{aligned}
p_{\mu} & \equiv \kappa \frac{P_{\mu}}{P_{4}}, \\
x_{\mu} & \equiv \frac{1}{\kappa} J_{\mu 4}=\frac{1}{\kappa}\left(X_{\mu} P_{4}-X_{4} P_{\mu}\right) .
\end{aligned}
$$

It is straightforward to check that these coordinates commute with both $\mathcal{H}_{5 d}$ and $\mathcal{C}=\mathcal{D}-T$. In terms of these $4 \mathrm{~d}$ variables, the kinetic part of $S_{5 d}$ can be written up to boundary terms as

$$
\dot{X}_{A} P^{A}=\dot{x}_{\mu} p^{\mu}-p_{\mu} \frac{x \cdot p}{\kappa^{2}-p^{2}} \dot{p}^{\mu},
$$

where in addition to the usual $\dot{x}_{\mu} p^{\mu}$ term, there is also a new term that depends on $\kappa$. Note that to arrive at the above formula, we explicitly imposed the constraint $\mathcal{H}_{5 d}=0$. 
Now, it is easy to compute the Poisson bracket of the $4 \mathrm{~d}$ variables either directly from the definitions (23) and the $5 \mathrm{~d}$ symplectic structure or from the kinematical part of the action given in (24). The resulting structure is

$$
\begin{aligned}
& \left\{x_{\mu}, x_{\nu}\right\}=-\frac{1}{\kappa^{2}} J_{\mu \nu}, \\
& \left\{x_{\mu}, p_{\nu}\right\}=\eta_{\mu \nu}-\frac{p_{\mu} p_{\nu}}{\kappa^{2}},
\end{aligned}
$$

where the Lorentz generators are:

$$
J_{\mu \nu}=\left(X_{\mu} P_{\nu}-X_{\nu} P_{\mu}\right)=\left(x_{\mu} p_{\nu}-x_{\nu} p_{\mu}\right) .
$$

This deformed symplectic structure defines the Snyder non-commutative space-time. When $\kappa$ goes to infinity, we recover the standard phase space of the relativistic particle.

Starting from (24), we note that the kinetic term is trivialized to $x_{\mu}^{\prime} \dot{p}^{\mu}$ with the new set of $4 d$ position coordinates:

$$
x_{\mu}^{\prime}=x_{\mu}+p_{\mu} \frac{x \cdot p}{\kappa^{2}-p^{2}} .
$$

The symplectic structure expressed with these new coordinates is simply $\left\{x_{\mu}^{\prime}, p_{\nu}\right\}=\eta_{\mu \nu}$. Since the new primed coordinates are defined only in terms of the unprimed ones, they still commute with both the Hamiltonian constraint and the gauge-fixing condition. The natural issue is the physical meaning and interpretation of these new coordinates $x_{\mu}^{\prime}$.

Since the $4 \mathrm{~d}$ variables $x_{\mu}$ and $p_{\mu}$ commute with the $5 \mathrm{~d} \kappa$-shell constraint $\mathcal{H}_{5 d}$, we can interpret $x, p$ as Dirac observables of the $5 \mathrm{~d}$ system (with respect to the $5 \mathrm{~d} \kappa$ shell constraint, not the $P_{4}=M$ mass-shell constraint). On the other hand, this means that considered as operators they leave the De Sitter space invariant: the $x_{\mu}$ 's generate the translations on the De Sitter space, which has become our $4 \mathrm{~d}$ momentum space. Since $x_{\mu}, p_{\mu}$ also commute with the gauge fixing condition $\mathcal{D}$, we can fix $\mathcal{H}_{5 d}=\mathcal{D}=0$ in the ten-dimensional phase space without interfering with the $x, p$ variables and reduce the phase space to eight-dimensions. By this, we mean that the gauge fixed bracket -the Dirac bracket- of $x_{\mu}, p_{\mu}$ with any function will be equal to their Poisson bracket. This also allows us to invert the definition (23) and express the 5 d coordinates $X, P$ in terms of $x, p, \kappa, T$. The condition $\mathcal{H}_{5 d}=0$ is used to obtain the 5 d momentum $P_{A}$, while the condition $\mathcal{D}=0$ gives the expression for the $5 \mathrm{~d}$ position $X_{A}$. We denote the resulting functions $\widetilde{X}$ and $\widetilde{P}$ in order to avoid confusion with the original variables $X, P$,

$$
\begin{aligned}
& \widetilde{P}_{4}=\frac{\kappa}{\sqrt{1-\frac{p^{2}}{\kappa^{2}}}}, \quad \widetilde{P}_{\mu}=\frac{p_{\mu}}{\sqrt{1-\frac{p^{2}}{\kappa^{2}}}}, \\
& \widetilde{X}_{4}=\frac{1}{\kappa \sqrt{1-\frac{p^{2}}{\kappa^{2}}}}(x \cdot p-T), \\
& \widetilde{X}_{\mu}=x_{\mu} \sqrt{1-\frac{p^{2}}{\kappa^{2}}}+\frac{p_{\mu}}{\kappa^{2} \sqrt{1-\frac{p^{2}}{\kappa^{2}}}}(x \cdot p-T),
\end{aligned}
$$

or in a more condensed format:

$$
\begin{aligned}
& \widetilde{X}_{\mu}=\frac{\kappa}{\widetilde{P}_{4}} x_{\mu}^{\prime}-T \frac{\widetilde{P}_{\mu}}{\kappa}, \\
& \widetilde{X}_{4}=\frac{\widetilde{P}_{4}}{\kappa^{2}} x \cdot p-T \frac{\widetilde{P}_{4}}{\kappa} .
\end{aligned}
$$

On the one hand, this explains the origin of the $x_{\mu}^{\prime}$ coordinates: they are the four-dimensional sector of the $5 \mathrm{~d}$ coordinates (at $T=0)$. On the other hand, the inversion formula reveals the meaning of the fifth space-time coordinate (in the Snyder basis): it is the dilatation $D=x . p$ on the 4-dimensional relativistic particle.

Finally, we can compute the Dirac bracket corresponding to our gauge fixing. We obtain:

$$
\begin{aligned}
\left\{X_{A}, X_{B}\right\}_{D} & =\frac{-1}{P_{C} P^{C}} J_{A B}=\frac{1}{\kappa^{2}} J_{A B}, \\
\left\{X_{A}, P_{B}\right\}_{D} & =\eta_{A B}-\frac{P_{A} P_{B}}{P_{C} P^{C}}=\eta_{A B}+\frac{P_{A} P_{B}}{\kappa^{2}} .
\end{aligned}
$$

First, we point out that the Dirac bracket of the $4 \mathrm{~d}$ variables $x_{\mu}, p_{\mu}$ is equal to the Poisson bracket since they commute with $\mathcal{H}_{5 d}$ : the bracket is unchanged and we remain with the commutation relations (25). Second, we have the following relations on the $5 \mathrm{~d}$ variables:

$$
\begin{aligned}
\left\{\widetilde{X}_{A}, \widetilde{X}_{B}\right\} & =\left\{X_{A}, X_{B}\right\}_{D} \\
\left\{\widetilde{X}_{A}, \widetilde{P}_{B}\right\} & =\left\{X_{A}, P_{B}\right\}_{D}, \\
\left\{\widetilde{P}_{A}, \widetilde{P}_{B}\right\} & =\left\{P_{A}, P_{B}\right\}_{D}
\end{aligned}
$$

\section{B. 4d DSR Physics}

So far, we have derived the $4 \mathrm{~d}$ phase space by gauge fixing the $5 \mathrm{~d}$ phase space. Now we are interested in the dynamics of the resulting $4 \mathrm{~d}$ particle. By this we mean writing down the $4 \mathrm{~d}$ Hamiltonian flow, choosing a time variable and the equations of motion. 
The fifth component of momentum $P_{4}$ is equal to $\sqrt{\kappa^{2}-P_{\mu} P^{\mu}}$ on the $\kappa$-shell and is a $4 \mathrm{~d}$ Lorentz invariant. Moreover, as $P_{4}=\kappa / \sqrt{1-p^{2} / \kappa^{2}}$ is simply a function of $p^{2}$, we can make the $4 \mathrm{~d}$ Hamiltonian constraint $\mathcal{H}_{4 d}=P_{4}-M$ of the same form as (4), i.e.

$$
\mathcal{H}_{4 d}=p^{2}-m^{2}=0,
$$

with

$$
M=\frac{\kappa}{\sqrt{1-\frac{m^{2}}{\kappa^{2}}}}, \quad m=\kappa \sqrt{1-\frac{\kappa^{2}}{M^{2}}} .
$$

Note that the rest mass $m$ is bounded by the universal mass scale $\kappa$, while $M$ is necessarily larger than $\kappa$. Computing the Hamiltonian flow of the position variables gives

$$
\delta x_{\mu}=\left\{\lambda \mathcal{H}, x_{\mu}\right\}=-2 \lambda p_{\mu}\left(1-\frac{p^{2}}{\kappa^{2}}\right),
$$

leading to the same speed formula $v_{i} \equiv \dot{x}_{i}=p_{i} / p_{0}$ as in special relativity if we choose $t=x_{0}$ as our time. Therefore, we have the same relation between the speed and momentum as in special relativity, $p_{\mu}=\left(\gamma m, \gamma m v_{i}\right)$.

The similarity between Snyder basis physics and standard special relativity suggests that there should be a direct mapping between the two. Indeed one can introduce commutative coordinates $y_{\mu}, q_{\mu}$ in the Snyder basis $x_{\mu}, p_{\mu}$. Let

$$
\begin{aligned}
& y_{\mu}=\kappa \frac{x_{\mu}}{\widetilde{P}_{4}}=x_{\mu} \sqrt{1-\frac{p^{2}}{\kappa^{2}}}, \\
& q_{\mu}=\frac{\widetilde{P}_{4} p_{\mu}}{\kappa}=\frac{p_{\mu}}{\sqrt{1-\frac{p^{2}}{\kappa^{2}}}} .
\end{aligned}
$$

Then these new coordinates satisfy trivial canonical relations

$$
\left\{y_{\mu}, y_{\nu}\right\}=0, \quad\left\{y_{\mu}, q_{\nu}\right\}=\eta_{\mu \nu} .
$$

The Hamiltonian flow on the phase space $(y, q)$ is equivalently generated by $p^{2}$ or $q^{2}$. Thus everything looks like we had rescaled the standard flat metric $\eta_{\mu \nu}$ of the Minkowski by a momentum-dependent factor $\left(1-p^{2} / \kappa^{2}\right)$. This type of momentum-dependent metric naturally appearing in the Snyder basis allow an explicit link with the rainbow metric formalism advocated in [22]. When its rest mass $m$ reaches the maximal bound $\kappa$, the metric $d x^{2}=d y^{2} /\left(1-p^{2} / \kappa^{2}\right)$ goes to infinity. In some sense, when $m=\kappa$, the particle freezes -it doesn't move. Finally the gauge fixed $5 \mathrm{~d}$ variables have a simple expression in terms of $y$ and $q$ :

$$
\widetilde{P}_{\mu}=\pi_{\mu}, \quad \widetilde{X}_{\mu}=y_{\mu}+\frac{q_{\mu}}{\kappa^{2}}(y \cdot q-T) .
$$

The Dirac observables for the DSR particle are simply $p_{\mu}$ (or the rescaled $q_{\mu}$ ) and $J_{\mu \nu}$, which generates the usual (undeformed) Poincaré algebra. One can construct position Dirac observables the same way as done for the undeformed relativistic particle. Indeed, despite the deformed symplectic structure, the position operators $x_{\mu}^{(D)}=x_{\mu}+p_{\mu} / p^{2}(T-x \cdot p)$, with an arbitrary parameter $T$, still commute with the Hamiltonian constraint $p^{2}$ and are therefore Dirac observables.

From the 5d perspective, it is even more straightforward to obtain such position Dirac observables commuting with both constraints, $\mathcal{H}_{5 d} \sim P_{A} P^{A}$ and $\mathcal{H}_{4 d} \sim P_{4}$. Indeed, the positions $X_{\mu}$ commute with $P_{4}$. Nevertheless these variables do not commute with the $\kappa$-shell condition $P_{A} P^{A}=-\kappa^{2}$. We can still use the same construction as for the $4 \mathrm{~d}$ relativistic particle applied to our $5 \mathrm{~d}$ particle and write down position Dirac observables on De Sitter as $X_{\mu}+P_{\mu} / P^{2}(T-X . P)$. These actually are the previous $x_{\mu}^{(D)}$ re-scaled by $\kappa / P_{4}$.

\section{Relativistic effects?}

$L^{2} \equiv x_{\mu} x^{\mu}$ is the natural Lorentz invariant quadratic function of the Snyder basis positions $x_{\mu}$ and will be therefore considered as defining the Minkowski metric in our non-commutative space-time. In terms of the $5 \mathrm{~d}$ variables, it reads as:

$$
\begin{aligned}
\kappa^{2} L^{2} & =J_{4 \mu} J^{4 \mu} \\
& =P_{4}^{2} X_{\mu} X^{\mu}+X_{4}^{2} P_{\mu} P^{\mu}-2 X_{4} P_{4} X_{\mu} P^{\mu} .
\end{aligned}
$$

In the Snyder basis, the metric is unchanged, the action of the Lorentz transformations on $(x, p)$ is as unmodified and the speed-momentum relation is the same as in special relativity, therefore we do not expect any DSR effect on the motion of the single DSR relativistic particle. The spectrum of the $L^{2}$ at the quantum level can be found in [23].

We expect DSR effects to appear for multi-particle systems through a modification of the energy-momentum conservation and deformed scattering amplitudes. This should be connected to the fact that there does not exist any non-trivial deformation of the Poincaré symmetry as a Lie algebra, but there does exist non-trivial deformations as a Hopf algebra structure: $\kappa$-deformed Poincaré can always be re-written as the standard Poincaré Lie algebra while all the information about the deformation will be contained in the co-product i.e the laws of addition and conservation of energy-momentum.

A last comment is that if we allow position-momentum mixing in the length operator and therefore momentumdependent metrics, we have access to Lorentz invariants 
other than $x_{\mu} x^{\mu}$, such as the rest mass $p^{2}$ and the dilatation x.p. For instance, the 5 d invariant metric has an interesting expression in terms of the $4 \mathrm{~d}$ variables:

$$
\begin{aligned}
\widetilde{X}_{A} \widetilde{X}^{A} & =x_{\mu} x^{\mu}\left(1-\frac{p^{2}}{\kappa^{2}}\right)+\frac{T(x \cdot p-T)}{\kappa^{2}}, \\
& =y_{\mu} y^{\mu}+\frac{T(y \cdot q-T)}{\kappa^{2}} .
\end{aligned}
$$

On the $5 \mathrm{~d}$ light-cone, $T=X_{A} P^{A}=0$, this metric reduces to the rescaled flat metric which is thus the natural Lorentz invariant metric from the $5 \mathrm{~d}$ perspective. Nevertheless, the physical interpretation of this metric at $T \neq 0$ is not yet clear to us. For traditional $4 \mathrm{~d}$ physics, $T$ does not evolve during the free motion of the system. This could nevertheless be affected by the introduction of a non-trivial dynamics (through forces). See however the discussion of the metric in the bicrossproduct basis under equation (52) below.

\section{BICROSSPRODUCT BASIS}

To obtain the bicrossproduct version of DSR, we choose the gauge fixing condition $\mathcal{C}$ as

$$
\mathcal{C}=\frac{X_{0}-X_{4}}{P_{0}-P_{4}}-T
$$

where $T$ is again a free parameter. Therefore the bicrossproduct basis is a light cone gauge for the $5 \mathrm{~d}$ action.

As in the previous section, we first study the reduced phase space obtained by implementing this constraint, and then look at various features of the resulting $4 \mathrm{~d}$ physics.

\section{A. Reduced phase space}

A set of $4 \mathrm{~d}$ momentum variables that commute with both $\mathcal{H}_{5 d}$ and $\mathcal{C}$ are

$$
p_{0} \equiv \kappa \ln \frac{P_{4}-P_{0}}{\kappa}, \quad p_{i} \equiv \frac{\kappa P_{i}}{P_{0}-P_{4}} .
$$

As for the position variables, we choose

$$
x_{0} \equiv \frac{1}{\kappa} J_{40}, \quad x_{i} \equiv \frac{1}{\kappa}\left(J_{i 0}-J_{i 4}\right) .
$$

The ten variables $\left(\mathcal{H}_{5 d}, \mathcal{C}, x_{\mu}, p_{\mu}\right)$ parametrize the tendimensional phase space. Actually, we are restricted to the sector $P_{4}>P_{0}$. If we want to parametrize the whole space, we should allow sign changes in the definition of the $4 \mathrm{~d}$ momentum $p_{\mu}$.
From here, we repeat calculations similar to those presented in the previous section on the Snyder gauge-fixing. When we impose $P_{A} P^{A}+\kappa^{2}=0$, the kinetic part of the $5 \mathrm{~d}$ action reduces to

$$
\dot{X}_{A} P^{A}=p_{\mu} \dot{x}_{\mu}+p_{i} x_{i} \dot{p}_{0} .
$$

This provides the $4 \mathrm{~d}$ action principle describing the DSR particle in the bicrossproduct basis. This kinetic term directly gives the $\kappa$-Minkowski symplectic structure on the $4 \mathrm{~d}$ phase space,

$$
\begin{aligned}
& \left\{x_{0}, p_{0}\right\}=1, \quad\left\{x_{i}, p_{j}\right\}=-\delta_{i j}, \\
& \left\{x_{0}, x_{i}\right\}=+\frac{1}{\kappa} x_{i}, \quad\left\{x_{0}, p_{i}\right\}=-\frac{1}{\kappa} p_{i},
\end{aligned}
$$

with all other brackets vanishing.

A similar 4d action was proposed in [24] as describing a particle propagating in the $A d S$ space-time. The $4 \mathrm{~d}$ Lagragian was defined in term of $\left(x_{\mu}, \dot{x}^{\mu}\right)$ with a $A d S$ metric and the Dirac brackets taking into account the 2nd class constraints were shown to reproduce the $\kappa$-Minkowski brackets. That action realizes the inverse Legendre transform of our Lagragian expressed in the variables $\left(x_{\mu}, p^{\mu}\right)$.

Solving the $5 \mathrm{~d}$ Hamiltonian constraint $\mathcal{H}_{5 d}=0$ and fixing the gauge at $\mathcal{C}=0$ allows to invert (34) and (35) to obtain

$$
\begin{aligned}
\widetilde{P}_{0} & =-\kappa \sinh \frac{p_{0}}{\kappa}-\frac{\vec{p}^{2}}{2 \kappa} e^{\frac{p_{0}}{\kappa}}, \\
\widetilde{P}_{i} & =-p_{i} e^{\frac{p_{0}}{\kappa}}, \\
\widetilde{P}_{4} & =\kappa \cosh \frac{p_{0}}{\kappa}-\frac{\vec{p}^{2}}{2 \kappa} e^{\frac{p_{0}}{\kappa}}, \\
\widetilde{X}_{\mu} & =\kappa \frac{x_{\mu}}{P_{0}-P_{4}}+T P_{\mu}, \\
\widetilde{X}_{4} & =\kappa \frac{x_{0}}{P_{0}-P_{4}}+T P_{4} .
\end{aligned}
$$

The first set of relations are just the familiar definitions of the bicrossproduct basis in terms of planar coordinates on de Sitter.

The Dirac bracket induced by the gauge fixing condition acts on the $5 \mathrm{~d}$ variables as

$$
\begin{aligned}
\left\{X_{0}, X_{4}\right\}_{D} & =\frac{1}{2} \frac{X_{0}-X_{4}}{P_{0}-P_{4}}, \\
\left\{X_{i}, P_{j}\right\}_{D} & =\delta_{i j}, \\
\left\{X_{0}, P_{0}\right\}_{D} & =-1-\frac{1}{2} \frac{P_{0}}{P_{0}-P_{4}}, \\
\left\{X_{4}, P_{4}\right\}_{D} & =+1-\frac{1}{2} \frac{P_{4}}{P_{0}-P_{4}}, \\
\left\{X_{0}, P_{4}\right\}_{D} & =-\frac{1}{2} \frac{P_{0}}{P_{0}-P_{4}}, \\
\left\{X_{4}, P_{0}\right\}_{D} & =-\frac{1}{2} \frac{P_{4}}{P_{0}-P_{4}} .
\end{aligned}
$$


As earlier, these important facts about the Dirac bracket are that, first, the Dirac bracket of $(x, p)$ with any other phase space function is exactly equal to the Poisson bracket and, second, the Poisson brackets of the $(\widetilde{X}, \widetilde{P})$ variables is equal to the Dirac bracket of the $(X, P)$ variables computed above.

As in the Snyder case, we can find an alternate system of position variables that trivializes the $4 d$ symplectic structure. For the bicrossproduct basis, we achieve this by defining

$$
x_{0}^{\prime}=x_{0}-\frac{1}{\kappa} x_{i} p^{i}, \quad \text { and } \quad x_{i}^{\prime}=x_{i} .
$$

This choice of space-time coordinates is commutative and the couple $\left(x^{\prime}, p\right)$ have canonical Poisson brackets. In terms of the $5 \mathrm{~d}$ coordinates, $x_{0}^{\prime}$ is given by

$$
\begin{aligned}
\kappa x_{0}^{\prime} & =J_{40}-\frac{P_{i}}{P_{0}-P_{4}}\left(J_{i 0}-J_{i 4}\right) \\
& =\left(X_{4} P_{0}-X_{0} P_{4}\right)-X_{i} P_{i}+P_{i} P_{i} \frac{X_{0}-X_{4}}{P_{0}-P_{4}} .
\end{aligned}
$$

This variable is singular at $P_{0}-P_{4}=0$. In terms of the $4 \mathrm{~d}$ momentum coordinates, this singularity happens for infinite "energy", $p_{0}=\infty$. The $P_{0}-P_{4}=0$ is also the singularity for the bicrossproduct momentum variables (34). Therefore one could decide that the coordinates $x^{\prime}$ are better mathematical space-time coordinates and that they should be the physical space-time coordinates since they do commute. However, as we will see below, the speed of a DSR particle in the $x^{\prime}$ basis is not bounded by the speed of light $c$ while the speed computed in the $x$ basis will be bounded by $c$ and behave appropriately.

\section{B. 4d physics}

We now turn to the physics and dynamics of the DSR particle in the bicrossproduct basis.

The 4 d Hamiltonian constraint $\mathcal{H}_{4 d}=P_{4}-M$ can be re-written as:

$$
\begin{gathered}
\mathcal{H}_{4 d}=\frac{1}{2 \kappa} \mathcal{H}+\kappa-M, \\
\mathcal{H}=\left(2 \kappa \sinh \frac{p_{0}}{2 \kappa}\right)^{2}-\vec{p}^{2} e^{\frac{p_{0}}{\kappa}},
\end{gathered}
$$

where $\mathcal{H}$ goes to $p^{2}$ in the classical limit $\kappa \rightarrow \infty$. $\mathcal{H}$ is the Casimir of the $\kappa$-deformed Poincaré group in the bicrossproduct basis [25].

$\mathcal{H}_{4 d}=0$ reduces to the mass-shell condition $\mathcal{H}=m^{2}$ for the rest mass:

$$
m^{2}=2 \kappa(M-\kappa),
$$

where $M$ is restricted to be larger than $\kappa$.

The mass-shell condition relates the space moment $\vec{p}^{2}$ to the energy $p_{0}$ as:

$$
\vec{p}^{2}=e^{-\frac{p_{0}}{\kappa}}\left(2 \kappa^{2} \cosh \frac{p_{0}}{\kappa}-2 \kappa^{2}-m^{2}\right) .
$$

At zero speed, $\vec{p}=0$, it is possible to invert the cosh and, assuming that $p_{0}$ is the (measured) energy $E$, we obtain corrections to the $E=m c^{2}$ formula:

$$
\begin{aligned}
p_{0} & =2 \kappa \ln \left(\frac{m}{2 \kappa}+\sqrt{1+\frac{m^{2}}{4 \kappa^{2}}}\right), \\
& =\kappa \ln \left(1+\frac{m}{\kappa} \sqrt{1+\frac{m^{2}}{4 \kappa^{2}}}+\frac{m^{2}}{2 \kappa^{2}}\right), \\
& \sim m-\frac{1}{24} \frac{m^{3}}{\kappa^{2}}+\ldots
\end{aligned}
$$

A measurable DSR effect would then be an energy default with the rest energy being actually smaller than the mass $m$. Nevertheless, this relies on two assumptions. The first one is that $p_{0}$ is effectively the energy that we measure. More generally, we should carefully define the energy-momentum $p_{\mu}$ operationally as measurements. As an example, in 3d quantum gravity, the momentum is defined as a measurement of the geometry through the holonomy around the particle [7]. The second issue is that $m$ might not be the true mass, but simply the bare gravitational mass. Then the mass defined above could be the renormalized mass taking into account the gravitational self-energy i.e the one that we do measure in a real experiment. This phenomenon happens in $3 \mathrm{~d}$ quantum gravity where the bare mass $m$ (creating the conical singularity defining the particle) gets renormalized to $\kappa \sin (m / \kappa)$ due to gravitational effect [7]. Such effects are also expected in $4 \mathrm{~d}$ gravity [28].

The momentum light cone is defined as the limit case of a massless particle $m=0$. In that case, the dispersion relation simplifies to:

$$
|\vec{p}|=\kappa\left(1-e^{-\frac{p_{0}}{\kappa}}\right), \quad p_{0}=-\kappa \ln \left(1-\frac{|\vec{p}|}{\kappa}\right) .
$$

For an arbitrary mass, it is still possible to extract the energy in term of the spatial momentum. For computation purpose, it is convenient to introduce $\alpha \equiv \exp \left(p_{0} / \kappa\right)$ or equivalently $p_{0}=\kappa \ln \alpha$. Then the dispersion relation is a second degree polynomial in $\alpha$ and can be solved explicitly to:

$$
\begin{gathered}
\alpha_{ \pm}=\frac{\left(m^{2}+2 \kappa^{2}\right) \pm \sqrt{\Delta}}{2\left(\kappa^{2}-\vec{p}^{2}\right)}, \\
\Delta \equiv m^{4}+4 m^{2} \kappa^{2}+4 \vec{p}^{2} \kappa^{2} .
\end{gathered}
$$


It is straightforward to take the classical limit $\kappa \rightarrow+\infty$ where we are supposed to recover undeformed special relativity:

$$
\alpha_{ \pm}=e^{\frac{p_{0}}{\kappa}} \sim 1+\frac{p_{0}}{\kappa} \sim 1 \pm \frac{1}{\kappa} \sqrt{\vec{p}^{2}+m^{2}},
$$

which allows us to identify $\alpha_{ \pm}$as the positive and negative energy modes. Let us underline that the negative energy mode is not given by $p_{0} \rightarrow-p_{0}$. This is reminiscent of the deformation of the antipode in the quantum group language.

The equations of motions arising from the flow with respect to $\lambda \mathcal{H}$ are

$$
\begin{aligned}
& \delta x_{0}=-2 \lambda\left(\kappa \sinh \frac{p_{0}}{\kappa}+\frac{\vec{p}^{2}}{2 \kappa} e^{\frac{p_{0}}{\kappa}}\right), \\
& \delta x_{i}=-2 \lambda p_{i} e^{\frac{p_{0}}{\kappa}} \\
& \delta p_{0}=\delta p_{i}=0 .
\end{aligned}
$$

If we choose $t=x_{0}$ as the time variable, then the speed is defined as usual as $v_{i} \equiv \delta x_{i} / \delta x_{0}$. In this case, we can compute the norm of $v_{i}$ in terms of $p_{0}$,

$$
v^{2}=\frac{e^{2 \zeta}-u e^{\zeta}+1}{e^{2 \zeta}-u e^{\zeta}+\left(\frac{u}{2}\right)^{2}},
$$

which we express in term of the dimensionless energy $\zeta \equiv$ $p_{0} / \kappa$ and dimensionless mass $u \equiv m^{2} / \kappa^{2}+2$. It is clear that the speed is still bounded by the speed of light $c=1$ (more exactly, the speed of massless particles). Moreover as in standard physics, the speed get reach $c$ if and only if $u=2$ i.e the mass vanishes $m=0$. Such a relation $v\left(p_{0}\right)$ could be checked experimentally, assuming than $p_{0}$ is the energy.

To complete the analysis, it is interesting to invert the relation between the speed and the momentum in order to get the deformed equivalent of the standard speedmomentum relation $p_{\mu}=(\gamma m, \gamma m \vec{v})$. First we get the energy:

$$
\alpha_{ \pm}(v)=1 \pm \gamma \sqrt{\frac{m^{2}}{\kappa^{2}}+\frac{m^{4}}{4 \kappa^{4}}}+\frac{m^{2}}{2 \kappa^{2}},
$$

where we keep the standard definition $\gamma \equiv 1 / \sqrt{1-v^{2}}$. In the classical limit $\kappa \rightarrow+\infty$, we recover $p_{0} \sim \pm \gamma m$. Then we can compute the spatial moment as:

$$
\vec{p}=\vec{v}\left(\kappa-\frac{m^{2}+2 \kappa^{2}}{2 \kappa} e^{-\frac{p_{0}}{\kappa}}\right)=\vec{v}\left(\kappa-\frac{m^{2}+2 \kappa^{2}}{2 \kappa \alpha}\right) .
$$

At zero speed $\vec{v}=0$, the momentum $\vec{p}$ vanishes as expected. But the proportionality coefficient is deformed, it is not simply the mass $m$ but it has a more complicated form:

$$
\vec{p} \underset{v \rightarrow 0}{\sim} \vec{v} \frac{m \sqrt{1+\frac{m^{2}}{4 \kappa^{2}}}}{1+\frac{m^{2}}{2 \kappa^{2}}+\frac{m}{\kappa} \sqrt{1+\frac{m^{2}}{4 \kappa^{2}}}} .
$$

On the other side, in the limit $v \rightarrow c$ where the speed reaches the speed of light, the momentum does not diverge as in special relativity and we find that $\vec{p}=\kappa \vec{v}$ and we have a finite effective mass $|\vec{p}| /|\vec{v}|$.

We conclude this analysis with the remark that if we had chosen $t=x_{0}^{\prime}$ as time, then we could have done the exact same calculations but we would have found that the speed with respect to $x_{0}^{\prime}$ would increase exponentially with $p_{0}$ and diverge to infinity. This is to be compared to the use of the dilatation time $D / m$ in special relativity. Therefore, we discard it as a physical time coordinate since we want to keep a maximal speed bound defined by the speed of light $c$.

The symmetry generators (19), in terms of $p_{\mu}$ and $x_{\mu}$ coordinates, are

$$
\begin{aligned}
M_{i j} & =J_{i j}=x_{i} p_{j}-x_{j} p_{i}, \\
N_{i} & =J_{i 0}=x_{i}\left(\kappa e^{-\frac{p_{0}}{\kappa}} \sinh \frac{p_{0}}{\kappa}+\frac{\vec{p}^{2}}{2 \kappa}\right)-x_{0} p_{i} .
\end{aligned}
$$

The rotations are undeformed, and the boosts are deformed in the first term. Nevertheless, they still form an undeformed Lorentz algebra (for the deformed symplectic structure). However, their action on the space-time coordinates will be deformed. More precisely, we compute their action on the position variables $x_{\mu}$ :

$$
\begin{aligned}
\left\{N_{i}, x_{0}\right\} & =x_{i}-\frac{1}{\kappa} N_{i}, \\
\left\{N_{i}, x_{j}\right\} & =x_{0} \delta_{i j}-\frac{1}{\kappa} M_{i j}, \\
\left\{M_{i j}, x_{0}\right\} & =0 \\
\left\{M_{i j}, x_{k}\right\} & =x_{j} \delta_{i k}-x_{i} \delta_{j k} .
\end{aligned}
$$

The action of these generators on the momentum variables gives the well known bicrossproduct relations. Overall, the rotation algebra does not seem deformed whatsoever. On the other hand, the boost sector is different than usual; we therefore study this sector in more details below and compute the effect of this boost deformation on the traditional length contraction.

We conclude this subsection with a discussion of the Lorentz invariant quadratic form, the "metric", in the bicrossproduct spacetime. In this basis, $x_{\mu} x^{\mu}$ is not a Lorentz invariant anymore. However we can deform it into

$$
L^{2}=x_{0}^{2}-\left(x_{i}-\frac{1}{\kappa} N_{i}\right)^{2},
$$


which can be thought of in principle as a new metric. This expression is exactly the same algebraic quantity as the Snyder basis metric, $J_{4 \mu} J^{4 \mu}$ in term of the 5 d operators. However, since the quantity $N_{i}$ depends on the particle momentum, we obtain a momentum-dependent quadratic form, explicitly:

$$
\begin{gathered}
L^{2}=x_{0}^{2}\left(1-\frac{\vec{p}^{2}}{\kappa^{2}}\right)-x_{i} x_{i} f(p)^{2}-\frac{2 x_{0}}{\kappa} \vec{x} \cdot \vec{p} f(p), \\
f(p)=1-e^{-\frac{p_{0}}{\kappa}} \sinh \frac{p_{0}}{\kappa}-\frac{\vec{p}^{2}}{2 \kappa^{2}} .
\end{gathered}
$$

It is tempting to interpret the quadratic form (53) as a space time metric ${ }^{1}$.

The momentum-dependence in the metric is strange from the point of view of special relativity, but it would be much more natural from the general relativity perspective, where we expect the particle to deform the geometry of the surrounding space-time depending on its energymomentum tensor. This effect may still be present in the DSR limit of gravity.

This would mean that the spacetime metric would vary for different particles which is rather hard to accept physically. In fact, the metric usually has a clear operational meaning, being a measure of distances between spacetime points. What one should presumably do is to try to construct the metric operationally, in terms of physical rods and clocks, using the fact that, as we said above, one has in disposal a universal observer-independent scale of velocity. It is crucial that all inertial observers could agree on a way the metric is constructed, and that means exist to synchronize clocks and rods of observers at different points and/or moving with respect to each other with constant speed. We believe that the kinematical description of the particle presented here is a good starting point for such a construction.

\section{Relativistic effects}

We integrate explicitly the action of the boosts on space-time coordinate and use this computation to study the time dilatation and length contraction in the bicrossproduct basis.

Consider for simplicity the action of boosts along the $i=1$ direction. Defining $\left\{N_{1}, F\right\}=\partial_{\xi} F$ for any function

${ }^{1}$ Another natural quadratic form can be derived from the $5 \mathrm{~d}$ metric:

$$
\tilde{X}_{A} \tilde{X}^{A}=T \kappa x_{0}^{\prime}-x_{i} x_{i} e^{-2 \frac{p_{0}}{\kappa}}-T^{2} \kappa^{2} .
$$

However, the physical meaning of $T$ is not clear and the behavior of $\widetilde{X}_{A} \widetilde{X}^{A}$ under boosts is not simple.
$F$, we obtain from the commutators (52) the differential equations

$$
\begin{aligned}
\partial_{\xi} x_{0}(\xi) & =x(\xi)-N_{1}(\xi), \\
\partial_{\xi} x_{1}(\xi) & =x_{0}(\xi) \\
\partial_{\xi} x_{2}(\xi) & =-M_{3}(\xi) \\
\partial_{\xi} x_{3}(\xi) & =+M_{2}(\xi) .
\end{aligned}
$$

To solve this system of equations, it is worth simplifying the right hand sides. Since $\left\{N_{1}, N_{1}\right\}=0$, the object $N_{1}(\xi)$ in the first equation above is actually a constant independent of $\xi$. Therefore, $N_{1}(\xi)$ can be evaluated once at a certain time in one particular reference frame. Using the definition for $N_{1}$, one has

$$
n_{1}=\left[x_{1}\left(e^{-p_{0}} \sinh p_{0}+\frac{p^{2}}{2}\right)-x_{0} p_{1}\right]_{0}
$$

where the subscript 0 denotes that the expression is evaluated a set of initial conditions for $x_{\mu}, p_{\mu}$.

Another trick is to find the functions $M_{3}(\xi)$ and $M_{2}(\xi)$. This can be done by considering the set of differential equations

$$
\begin{aligned}
\partial_{\xi} M_{2}(\xi) & =N_{3}(\xi) \\
\partial_{\xi} N_{3}(\xi) & =M_{2}(\xi) \\
\partial_{\xi} M_{3}(\xi) & =-N_{2}(\xi) \\
\partial_{\xi} N_{2}(\xi) & =-M_{3}(\xi)
\end{aligned}
$$

whose solutions are

$$
\begin{aligned}
M_{2}(\xi) & =m_{2} \cosh \xi+n_{3} \sinh \xi \\
M_{3}(\xi) & =m_{3} \cosh \xi-n_{2} \sinh \xi \\
N_{2}(\xi) & =n_{2} \cosh \xi-m_{3} \sinh \xi \\
N_{3}(\xi) & =n_{3} \cosh \xi+m_{2} \sinh \xi .
\end{aligned}
$$

The coefficients all denote initial values.

These observations makes it straight-forward to write down the solutions to the system of differential equations (55):

$$
\begin{aligned}
& x_{0}(\xi)=x_{0}(0) \cosh \xi+x_{1}(0) \sinh \xi-n_{1} \sinh \xi \\
& x_{1}(\xi)=x_{0}(0) \sinh \xi+x_{1}(0) \cosh \xi-n_{1} \cosh \xi+n_{1} \\
& x_{2}(\xi)=x_{2}(0)-n_{2}+n_{2} \cosh \xi-m_{3} \sinh \xi \\
& x_{3}(\xi)=x_{3}(0)-n_{3}+n_{3} \cosh \xi+m_{2} \sinh \xi .
\end{aligned}
$$

Given the equations of motion (46), we can also study relativistic effects. The first of these is to check the dependence of the velocity $v_{i}=\delta x_{i} / \delta x_{0}$ on the boost parameter. Consider a particle that is initially at rest, i.e. its initial momentum $p_{i}=0$. Then, using the transformation laws of momenta $p_{\mu}[12]$, we find that

$$
v=\tanh \xi
$$


just like in special relativity. The result does not depend on the initial value of the particle's energy, thus we again find that all particles approach the speed of light $v=c=$ 1 after repeated boosts.

Other relativistic effects that are worth investigating are time dilation and length contraction. We follow the approach developed in [17] and consider a system of two particles. One particle, labeled $A$, is placed at $X^{A}=0$ and has zero momentum $P^{A}=0$ in some reference frame. The other particle, labeled $B$, is placed at $X^{B}=\ell$ and is also stationary $P^{B}=0$ in that reference frame. The equations of motion for these particles, in terms of their affine parameters $s_{A}$ and $s_{B}$, are

$$
\begin{aligned}
& X_{0}^{A}\left(s_{A}, \xi\right)=s_{A} \sinh p_{0}^{A} \cosh \xi \\
& X_{1}^{A}\left(s_{A}, \xi\right)=s_{A} \sinh p_{0}^{A} \sinh \xi \\
& X_{2}^{A}\left(s_{A}, \xi\right)=0 \\
& X_{3}^{A}\left(s_{A}, \xi\right)=0
\end{aligned}
$$

and

$X_{0}^{B}\left(s_{B}, \xi\right)=s_{B} \sinh p_{0}^{B} \cosh \xi+\ell \sinh \xi-n_{1}^{B} \sinh \xi$.

$X_{1}^{B}\left(s_{B}, \xi\right)=s_{B} \sinh p_{0}^{B} \sinh \xi+\ell_{1} \cosh \xi-n_{1}^{B} \cosh \xi+n_{1}^{B}$

$X_{2}^{B}\left(s_{B}, \xi\right)=\ell_{2}-n_{2}+n_{2} \cosh \xi$

$X_{3}^{B}\left(s_{B}, \xi\right)=\ell_{3}-n_{3}+n_{3} \cosh \xi$.

In one reference frame characterized by $\xi=0$, we define ‘time' along each particle's worldline by

$$
\tau=x_{0}\left(s^{\prime}, 0\right)-X_{0}(s, 0)
$$

as the difference of $x_{0}$ functions at different value of the affine parameter $s$. We can find how these quantities transform under boosts by introducing another frame with general $\xi$. Then time intervals becomes

$$
\tau^{\prime}=x_{0}\left(s^{\prime}, \xi\right)-x_{0}(s, \xi)=\tau \cosh \xi
$$

for each particle. This is time dilation in its standard (special relativistic) form.

We now discuss distance measurements. A naive way to define distance is to consider the coordinate difference

$$
d_{1}=x_{1}^{A}(0,0)-x_{1}^{B}(0,0)=\ell_{1}
$$

similarly expression in the other directions give $d_{2}$ and $d_{3}$. The distance interval between the particles in the second reference frame is computed by first setting $x_{0}^{A}\left(s_{A}, \xi\right)=$ $x_{0}^{B}\left(s_{B}, \xi\right)$ to obtain a relation between $s_{A}$ and $s_{B}$, and then doing the subtraction of coordinates. The result along the direction of the boost is

$$
\begin{aligned}
d_{1}^{\prime} & =x_{1}^{A}\left(s_{A}, \xi\right)-x_{1}^{B}\left(s_{B}, \xi\right) \\
& =\frac{1}{\cosh \xi} \ell_{1}+n_{1}^{B}\left(\frac{\cosh \xi-1}{\cosh \xi}\right) .
\end{aligned}
$$

The first term gives the usual length contraction effect, whereas the second term is a correction due to the momentum space curvature. Its significance grows with the original separation $\ell$ and with increasing $\xi$ values. Since $n_{1}^{B}$ is also a function of $p_{0}^{B}$, the correction is also dependant on what kind of particle is being discussed. In the orthogonal directions, the distances are just $d_{2}=x_{2}^{B}\left(s_{B}, \xi\right)$ and $d_{3}=x_{3}^{B}\left(s_{B}, \xi\right)$. In contrast to $d_{1}$, these distances become larger as the boost parameter is increased.

An alternative approach for looking at length contraction is to define distances in terms of the combination $x_{i}-N_{i}$, as suggested by the invariant interval (53). This amounts to a shift of the coordinates by a constant. Then, we have

$$
d_{i}=\left(x_{i}^{A}(0,0)-n_{i}^{A}\right)-\left(x_{i}^{B}(0,0)-n_{i}^{B}\right)=\ell_{i}-n_{i}^{B} .
$$

In a boosted frame, the new measured distance along the direction of the boost is

$$
d_{1}^{\prime}=\frac{1}{\cosh \xi} d_{1}
$$

the usual length contraction. In the other directions, however, there is an effect:

$$
d_{2}^{\prime}=d_{2}-n_{2}^{B}(1-\cosh \xi) .
$$

Directions orthogonal to the boost are therefore also slightly contracted.

\section{MENDES' BASIS}

Another basis was introduced by Vilela Mendes [26] in the context of the requirement of stability of the symmetry Lie algebra underlying physical systems. Concluding that the standard phase space structure underlying relativistic quantum mechanics (based on the Poincaré algebra) is not stable, he introduced a stable deformation, which can be represented in the extended ten-dimensional phase space.

The Mendes choice of $4 \mathrm{~d}$ coordinates is close to the Snyder basis but slightly different:

$$
p_{\mu}=P_{\mu}, \quad x_{\mu}=\frac{1}{\kappa} J_{\mu 4} .
$$

The $5 \mathrm{~d}$ kinetic term then reads

$$
-\dot{X}_{A} P^{A}=\frac{1}{P_{4}}\left(x_{\mu} \dot{p}^{\mu}\right)
$$

which is like the standard kinetic term rescaled by the momentum $P_{4}$. The Poisson brackets give the following commutators:

$$
\begin{aligned}
& \left\{x_{\mu}, x_{\nu}\right\}=-\frac{1}{\kappa^{2}} J_{\mu \nu} \\
& \left\{x_{\mu}, p_{\nu}\right\}=\eta_{\mu \nu} \frac{P_{4}}{\kappa}, \quad\left\{x_{\mu}, P_{4}\right\}=\frac{1}{\kappa} p_{\mu} .
\end{aligned}
$$


An important feature of these relations is that the scaling factor $P_{4}$ appears explicitly on the right hand side and must be treated as a new element of the algebra. In other words, the algebra of the variables $x_{\mu}$ and $p_{\mu}$ in this basis is not closed, so that the space spanned by these eight variables cannot be considered as the phase space of a $4 \mathrm{~d}$ spacetime independent of the fifth dimension.

Mathematically, the fact that the $x, p$ algebra is not closed means that there does not exist any gauge fixing condition leading to this choice of coordinates. Indeed, if we consider a phase space function $\mathcal{C}$ such that $\{\mathcal{C}, x\}=\{\mathcal{C}, p\}=0$, then we have $\left\{\mathcal{C}, P_{\mu}\right\}=\left\{\mathcal{C}, P_{4}\right\}=0$ since $\{x, p\} \sim P_{4}$. Thus $\mathcal{C}$ must commute with $\mathcal{H}_{5 d}$ and cannot be considered as a gauge fixing condition. Furthermore, since $\mathcal{C}$ must commute with $x_{\mu}=J_{\mu 4}, \mathcal{C}$ must be a $\mathrm{SO}(4,1)$-invariant function of only the $P_{A}$ 's. Thus it must be a function of $P_{A} P^{A}$.

We conclude that although Mendes' basis (67) amounts to a specific choice of parametrization of the tendimensional phase space, it does not allow a straightforward interpretation as a $4 \mathrm{~d}$ (non-commutative) spacetime through a gauge fixing of the $5 \mathrm{~d} \kappa$-shell constraint $\mathcal{H}_{5 d}$. Therefore, Mendes' basis is simply still a choice of variables for the same DSR theory, it does not allow the same interpretation as the Snyder basis or the bicrossproduct basis.

The other interesting feature of Mendes' work [26] is his prescription for cross-sections. He discusses a change of measure in momentum space:

$$
d^{3} p \rightarrow \frac{d^{3} p}{\sqrt{1-\frac{p^{2}}{\kappa^{2}}}} .
$$

On one hand, it is exactly the change of measure identified for 3d DSR [7] when considered as an effective theory for quantum gravity. On the other hand, it must be compared to the measure derived by Snyder for 4d DSR [1]:

$$
d^{4} p \rightarrow \frac{\kappa^{2} d^{4} p}{\kappa^{2}-p_{\alpha} p^{\alpha}} .
$$

Furthermore, Mendes postulates a trivial addition of momentum, $p_{\mu}^{\text {tot }}=p_{\mu}^{(1)}+p_{\mu}^{(2)}$. Since $p_{\mu}=P_{\mu}$, this fits with the proposal $[19,20]$ that the five-momentum $P_{A}$ is additive, but contradicts the standard view that the addition of $4 \mathrm{~d}$ momenta $p_{\mu}$ should be deformed.

\section{TOWARDS MANY-PARTICLE STATES}

In this paper, we have studied the dynamics of a single free particle in DSR. A necessary step towards building a quantum field theory in a DSR space-time is to study the classical mechanics of many particles, in particular two particles and the mechanics of the center of mass frame. A natural remark is that if we start from the $5 \mathrm{~d}$ action principle for the DSR particle, $\kappa$ does not need to be a universal mass scale but could be assumed to be a property of the considered particle on the same level than the rest mass $m$.

An important issue is the definitions of the addition of (energy)-momentum and of the total momentum in the center of mass frame. Usually, in DSR theories, one chooses a particular basis and writes down a deformed addition $p_{\mu} \oplus q_{\mu} \neq p_{\mu}+q_{\mu}$ such that the original momenta $p, q$ and the total momentum $p \oplus q$ are all on the same de Sitter space for the same fixed parameter $\kappa$. From the $5 \mathrm{~d}$ perspective, it is more natural to look for a prescription independent of any gauge fixing, and the most natural one is to simply add the $5 \mathrm{~d}$ momenta $P_{A}$. This is similar to when we go from Newtonian mechanics to special relativity. Indeed we straightforwardly add the new relativistic 4 -momenta. This does not simply reduce to a deformation of the 3-momentum addition since the addition of the (spatial) 3-momenta in special relativity involves explicitly the energies. In our context, let us define the total momentum in the center of mass frame as $R_{A}=P_{A}+Q_{A}$. Then the $\kappa$ parameter corresponding to the global two-particle system is

$$
\kappa_{\text {tot }}^{2}=-R_{A} R^{A}=\kappa_{1}^{2}+\kappa_{2}^{2}-2 P_{A} Q^{A} .
$$

In the special case when $P_{A}=Q_{A}$ and $\kappa_{1}=\kappa_{2}=\kappa$, the total $\kappa_{\text {tot }}$ is $2 \kappa$. This was actually already proposed in $[19,20]$ as a possible solution to the soccer ball problem in DSR.

Another related issue regards the relative system for the two particles. More precisely, one can look for the Lorentz invariant distance between two DSR particles. This distance should involve the energy-momentum of both particles, the same way that the metric in general relativity would depend on the energy-momentum tensor of both particles.

A more careful analysis would be required to understand the details of the separation of the two-particle system into global and relative systems.

\section{DISCUSSION}

In this paper, we have studied a classical particle in five space-time dimensions subject to two constraints defining two energy scales $m$ and $\kappa$. We have shown that, after gauge fixing, the $5 \mathrm{~d}$ model can give rise to various DSR models in $4 \mathrm{~d}$. The reduction from $5 \mathrm{~d}$ to $4 \mathrm{~d}$ selects a set of phase space coordinates $(x, p)$ via the requirement that they should commute with both the $\kappa$-shell constraint $\mathcal{H}_{5 d}$ and the gauge fixing function $\mathcal{C}$. 
We found that Snyder's basis uses the 5 d dilatation as its gauge fixing condition, while the bicrossproduct basis employs the 5d light-cone gauge. For both of these basis, we discussed the possible consequences of the scale $\kappa$ on $4 \mathrm{~d}$ physics. In principle, a "natural" gauge fixing $\mathcal{C}=X_{4}-T$ could also be studied. From the $5 \mathrm{~d}$ point of view, however, there is no clear reason to prefer one set of phase space coordinates over another one. This issue with coordinates is comparable to the one in General Relativity: General Relativity is diffeomorphism invariant and there is no canonical choice of "physical coordinates," although we always use a particular coordinate system to do explicit computations and write down the physical predictions.

In three space-time dimensions, the link between DSR and gravity has been clarified in [7]. Indeed, in 3d quantum gravity, particles are identified as conical singularities and their momentum is defined through non-local measurements as (a function of) the holonomy around the particle. This explicit characterization allows to rigorously derive DSR from 3d quantum gravity and unambiguously compute the Feynman diagrams for the resulting non-commutative quantum field theory [7].

There is also a proposal attempting to move the similarity between DSR and GR to the level of an explicit relationship in four dimensions [27]. In that proposal, the choice of coordinates $p_{\mu}$ (and $x_{\mu}$ ) correspond to the definition of the measured momenta (and positions) in terms of the tetrad field $e_{\mu}^{I}$. The issue then becomes: what are we exactly measuring physically when we talk bout the energy-momentum $p_{\mu}$ ? The answer to this question will determine the "correct" choice of physical coordinates to use in DSR. Regardless, we expect the physical predictions of DSR to be independent of any gauge fixing choice and propose that the "correctness" of a particular choice of coordinates should be measured by how convenient these coordinates are to express the measurements of a particular observer. For instance, one could try to properly define length measurements using clocks and time-of-flight experiments to define the metric operationally.

At the end of the day, we cannot make concrete predictions using DSR as long as we do not find gauge invariant quantities (commuting with the two constraints of the $5 \mathrm{~d}$ action) and their physical interpretation, or equivalently an explicit link between the choices of gauge fixing and measurement. This avenue of research seems to be a natural one from the $5 \mathrm{~d}$ perspective. It is also our view that the $5 \mathrm{~d}$ perspective should be a used when looking at twoparticle systems and studying their properties. Other related topics to be investigated are free spinning particles.

Finally, an important unresolved issue regards the physical interpretation of the fifth dimension. Written as a $5 \mathrm{~d}$ theory, DSR appears as a large extra dimension theory. We have proposed to see the coordinates in the fifth dimension as some effective degree of freedom coming from quantum gravity. The reformulation of GR as a $\mathrm{SO}(4,1) \mathrm{BF}$ gauge field theory proposed in [21] may prove to be a guide in this direction. It is also very tempting to interpret $P_{4}$ as the energy scale in a renormalisation scheme, as some kind of dynamical cut-off. $X_{4}$ would then be the generator of scale transformations. Such a speculation is supported by the fact that $X_{4}$ is (more or less) the $4 \mathrm{~d}$ dilatation operator in the Snyder basis, but this is truly little evidence. One could look at the renormalisation equation of a scalar field and try to interpret them as equations of motion in the DSR framework. The potential link between DSR and quantum gravity and the fact that the renormalisation flow of general relativity can be associated to a fifth dimension (with an AdS metric) [29] also points toward such an interpretation.

\section{Acknowledgments}

FG and EL would like to thank Laurent Freidel for many explanations on the Dirac observables for the relativistic particle. JKG wants to thank Perimeter Institute for hospitality in September 2005 when part of this work was completed. For JKG this work is partially supported by the KBN grant 1 P03B 01828. 
[1] H. Snyder, Quantized Space-Time, Phys.Rev. 71, 38 (1947).

[2] A. D. Donkov, R. M. Ibadov, V. G. Kadyshevsky, M. D. Mateev and M. V. Chizhov, Quantum Field Theory And A New Universal High-Energy Scale: Dirac Fields, Nuovo Cim. A 87 (1985) 373.

[3] A. D. Donkov, R. M. Ibadov, V. G. Kadyshevsky, M. D. Mateev and M. V. Chizhov, Quantum Field Theory And A New Universal High-Energy Scale. Gauge Vector Fields, Nuovo Cim. A 87 (1985) 350.

[4] V. G. Kadyshevsky and M. D. Mateev, Quantum Field Theory And A New Universal High-Energy Scale: The Scalar Model, Nuovo Cim. A 87 (1985) 324.

[5] S. Majid. Foundations of Quantum Groups Theory, Cambridge University Press.

[6] H. J. Matschull and M. Welling, Quantum mechanics of a point particle in 2+1 dimensional gravity, Class. Quant. Grav. 15 (1998) 2981 [arXiv:gr-qc/9708054].

[7] L. Freidel and E. R. Livine, Ponzano-Regge model revisited. III: Feynman diagrams and effective field theory, arXiv:hep-th/0502106.

[8] K. Imilkowska and J. Kowalski-Glikman, Doubly special relativity as a limit of gravity, arXiv:gr-qc/0506084.

[9] G. Amelino-Camelia, Testable scenario for relativity with minimum-length, Phys. Lett. B 510, 255 (2001) [arXiv:hep-th/0012238].

[10] G. Amelino-Camelia, Relativity in space-times with shortdistance structure governed by an observer-independent (Planckian) length scale, Int. J. Mod. Phys. D 11, 35 (2002) [arXiv:gr-qc/0012051].

[11] J. Kowalski-Glikman, Observer independent quantum of mass, Phys. Lett. A 286 (2001) 391 [arXiv:hepth/0102098].

[12] N. R. Bruno, G. Amelino-Camelia and J. KowalskiGlikman, Deformed boost transformations that saturate at the Planck scale, Phys. Lett. B 522 (2001) 133 [arXiv:hep-th/0107039].

[13] J. Magueijo and L. Smolin, Lorentz invariance with an invariant energy scale, Phys. Rev. Lett. 88 (2002) 190403 [arXiv:hep-th/0112090].

[14] J. Kowalski-Glikman, De Sitter space as an arena for doubly special relativity, Phys. Lett. B 547 (2002) 291 [arXiv:hep-th/0207279].

[15] J. Kowalski-Glikman and S. Nowak, Doubly special relativity and de Sitter space, Class. Quant. Grav. 20 (2003) 4799 [arXiv:hep-th/0304101].
[16] A. Blaut, M. Daszkiewicz, J. Kowalski-Glikman and S. Nowak, Phase spaces of doubly special relativity, Phys. Lett. B 582 (2004) 82 [arXiv:hep-th/0312045].

[17] J. Kowalski-Glikman, Planck-scale relativity from quantum kappa-Poincare algebra, Mod. Phys. Lett. A 17, 1 (2002) [arXiv:hep-th/0107054].

[18] L. Freidel, F. Girelli and E. R. Livine, Dirac observables and the Algebra of Deformed Special Relativity, in preparation

[19] F. Girelli and E. R. Livine, Physics of Deformed Special Relativity: Relativity Principle revisited, [arXiv:grqc/0412004].

[20] F. Girelli and E. R. Livine, Physics of Deformed Special Relativity, Braz.J.Phys. 35 (2005) 432-438, [arXiv:grqc/0412079].

[21] L. Freidel and A. Starodubtsev, Quantum gravity in terms of topological observables, [arXiv:hep-th/0501191].

[22] J. Magueijo and L. Smolin, Gravity's Rainbow, Class.Quant.Grav. 21 (2004) 1725-1736, [arXiv:grqc/0305055].

[23] E. R. Livine and D. Oriti, About Lorentz invariance in a discrete quantum setting, JHEP 0406 (2004) 050, [arXiv:gr-qc/0405085].

[24] S. Ghosh, The AdS particle, Phys.Lett. B623 (2005) 251257, [arXiv:hep-th/0506084].

[25] S. Majid and H. Ruegg, Bicrossproduct structure of kappa Poincare group and noncommutative geometry, Phys. Lett. B 334 (1994) 348, [arXiv:hep-th/9405107]; J. Lukierski, H. Ruegg and W. J. Zakrzewski, Classical quantum mechanics of free kappa relativistic systems, Annals Phys. 243 (1995) 90, [arXiv:hep-th/9312153].

[26] R. Vilela Mendes, Some consequences of a noncommutative space-time structure, Eur. Phys. J. C 42, 445 (2005) [arXiv:hep-th/0406013].

[27] R. Aloisio, A. Galante, A. Grillo, S. Liberati, E. Luzio and F. Mendez, Deformed Special Relativity as an effective theory of measurements on quantum gravitational backgrounds, [arXiv:gr-qc/0511031].

[28] J. D. Brown and J. W. York, Quasilocal energy and conserved charges derived from the gravitational action, Phys.Rev.D 47, 4 (1993).

[29] R. Percacci, The Renormalization Group, Systems of Units and the Hierarchy Problem, [arXiv: hepth/0409199]. 